IC/95/305, hep-th/9510067

International Atomic Energy Agency

and

United Nations Educational Scientific and Cultural Organization

INTERNATIONAL CENTRE FOR THEORETICAL PHYSICS

\title{
VACUUM POLARIZATION AND CHIRAL LATTICE FERMIONS
}

\author{
S. Randjbar-Daemi and J. Strathdee \\ International Centre for Theoretical Physics, Trieste, Italy.
}

\begin{abstract}
The vacuum polarization due to chiral fermions on a 4-dimensional Euclidean lattice is calculated according to the overlap prescription. The fermions are coupled to weak and slowly varying background gauge and Higgs fields, and the polarization tensor is given by second order perturbation theory. In this order the overlap constitutes a gauge invariant regularization of the fermion vacuum amplitude. Its low energy - long wavelength behaviour can be computed explicitly and we verify that it coincides with the Feynman graph result obtainable, for example, by dimensional regularization of continuum gauge theory. In particular, the Standard Model Callan-Symanzik RG functions are recovered. Moreover, there are no residual lattice artefacts such as a dependence on Wilson-type mass parameters.
\end{abstract}

\section{MIRAMARE - TRIESTE}

September 1995 


\section{Introduction}

The purpose of this paper is to supplement some earlier work on the "overlap prescription"[1] for describing chiral fermions on a Euclidean lattice. It was shown that this description is able to account for chiral anomalies in the coupling of lattice fermions to a slowly varying background gauge field and seems to give a correct formulation of the vacuum amplitude in perturbation theory [1-3]. The notorious problem [5] of fermion "doubling" is avoided in the overlap method and it has been verified that the low energy sector is indeed chiral.

A brief analysis of vacuum polarization due to chiral fermions was given in Ref.2. However, the discussion was incomplete in that a continuum approximation to the overlap prescription was used without a detailed justification. The consequent ultraviolet divergences were simply ignored. It is this aspect that will be dealt with here. In a lattice formulation there are of course no ultraviolet divergences. What is needed, therefore, is a somewhat more careful discussion of how the low energy physics is to be extracted from the lattice expressions. To illustrate how this goes, we consider here the computation of the fermionic contribution to the vacuum polarization tensor in a chiral gauge theory, including the effects of symmetry breaking due to a background Higgs field. We verify that the standard results of renormalized quantum field theory are obtained. In particular, we verify that there is no residual lattice effect such as a dependence on the Wilson mass parameter, $r$, an effect that has been of concern [6] to some other lattice models of chiral fermions [7].

The problem to be dealt with here, the computation of a vacuum polarization tensor, draws on work described in previous papers [1-3] 1. In particular, the idea of expressing the vacuum amplitude for chiral fermions coupled to background fields as the overlap of two distinct ground states is explained in great detail in these references. In Sec.2 we give only a brief summary of the overlap formalism in order to explain the notation and establish that the polarization tensor is gauge invariant even for finite lattice spacing. Some detail is provided in the Appendices. This should be contrasted with the gauge dependent result of [8] in the continuum 5-dimensional approach, where dimensional regularization was used.

The general formula for the polarization tensor is developed in Sec.2. It takes the form of a 4-dimensional Euclidean integral and represents a 1-loop effect. The region of integration is compact, a torus - or Brillouin zone - of volume $(2 \pi)^{4}$ in lattice units,

$$
\Pi_{\mu \nu}(k, \Lambda, \phi)=\int_{B Z}\left(\frac{d p}{2 \pi}\right)^{4} F_{\mu \nu}(p, k, \Lambda, \phi)
$$

\footnotetext{
${ }^{1}$ See also [4] where the vacuum polarization tensor has been discussed in a related context.
} 
where $\phi$ is a constant Higgs background and $\Lambda$ is an auxiliary parameter, a kind of regulator mass that plays a central role in the overlap formalism. The integrand, $F_{\mu \nu}$, is quite complicated and it is fortunate that most of its detailed structure is irrelevant to the low energy behaviour of $\Pi_{\mu \nu}$. What we need to understand is the behaviour near $k=\Lambda=\phi=0$. To see why this is so, consider for a moment the polarization tensor in arbitrary units where the lattice spacing, $a$, is not equal to unity. In such units the polarization tensor, $\Pi_{\mu \nu}^{\prime}$, which has dimension 2 , is given by

$$
\Pi_{\mu \nu}^{\prime}\left(k^{\prime}, \Lambda^{\prime}, \phi^{\prime}, a\right)=\frac{1}{a^{2}} \Pi_{\mu \nu}\left(a k^{\prime}, a \Lambda^{\prime}, a \phi^{\prime}\right)
$$

since $k^{\prime}, \Lambda^{\prime}$ and $\phi^{\prime}$ all have dimension 1 . Low energy means $k^{\prime}, \Lambda^{\prime}$ and $\phi^{\prime}$ are small relative to the lattice scale, $a^{-1}$. We are therefore required to consider the limit, $a \rightarrow 0$ with $k^{\prime}$, etc. fixed. Equivalently, in lattice units, we need to examine the behaviour of $\Pi_{\mu \nu}$ in the vicinity of $k=\Lambda=\phi=0$. It turns out, as will be discussed in Sec.3, that the integral (1.1) is singular at this point. This is an infrared singularity, of course, since the domain of integration is compact. In the vicinity of this point the integral is dominated by the threshold singularities associated with the vanishing of denominators in the expression for $F_{\mu \nu}(p, k, \Lambda, \phi)$ due to the propagation of chiral fermions near their mass shell.

To compute the dominant effects, i.e. the coefficients of the singular terms in $\Pi_{\mu \nu}$, we need to know the structure of $F_{\mu \nu}$ near $p=0$. In this region the lattice is irrelevant and we can safely approximate the integrand by "continuum" formulae, valid for $|p| \ll \pi$. We are tacitly assuming here that there are no other threshold singularities in (1.1) associated with massless fermion "doublers". It is necessary, of course, to make sure that such doublers do not in fact appear in the low energy fermion spectrum. The absence of such states is ensured in the overlap formalism by keeping the parameter, $\Lambda$, large relative to $k$ and $\phi$, but small relative to the lattice scale,

$$
k \sim \phi \ll \Lambda \ll 1
$$

The steps needed to isolate the infrared singularity in (1.1) are explained in some detail in Sec.3. The expression that emerges will then be recognized as the contribution of a conventional Feynman graph of renormalized continuum quantum field theory. In order to illustrate this equivalence we apply the formalism to Standard Model fermions, left handed doublets and right handed singlets, coupled to $S U(2) \times U(1)$ gauge fields and Higgs doublet (Sec.4). We calculate the Callan-Symanzik RG functions for quarks and leptons in the standard electroweak model.

In this paper we are exclusively concerned with the overlap approach to chiral gauge theories on the lattice. This approach grew out of earlier work by Kaplan [9] and others [10]. For some other approaches see [7, 11-14]. 


\section{The overlap prescription}

To obtained the vacuum amplitude for a collection of Weyl fermions coupled to background gauge and Higgs fields, one begins by doubling the number of degrees of freedom. Corresponding to each 2-component Weyl fermion introduce a 4-component Dirac field, $\psi(n)$, defined on a 4 -dimensional integer lattice, $n^{\nu} \in \mathbb{Z}^{4}$. The lattice is equipped with a Euclidean metric tensor, $g_{\mu \nu}$, invariant with respect to one of the 4 -dimensional crystal space groups. The choice of crystal structure is probably not very important but, in order to facilitate the emergence of $S O(4)$ invariance in the low energy sector, it is useful to employ a highly symmetric one such as the $F_{4}$ lattice [15]. For the $F_{4}$ lattice one can show, for example, that the polarization tensor is expressible in terms of two invariant functions,

$$
\Pi_{\mu \nu}(k)=\left(k^{2} g_{\mu \nu}-k_{\mu} k_{\nu}\right) \Pi_{1}+g_{\mu \nu} \Pi_{2}
$$

where $k^{2}=g^{\mu \nu} k_{\mu} k_{\nu}$ and $\Pi_{1}, \Pi_{2}$ are functions of $k^{2}$ and three other invariants of order 6 , 8 and 12 .

The fields $\psi(n)$ and $\psi(n)^{\dagger}$ satisfy anticommutation rules,

$$
\{\psi(n), \psi(m)\}=0, \quad\left\{\psi(n), \psi(m)^{\dagger}\right\}=\delta_{n m} 1, \quad\left\{\psi(n)^{\dagger}, \psi(m)^{\dagger}\right\}=0
$$

and they are used in the construction of two independent Hamiltonians,

$$
H_{ \pm}=\sum_{n, m} \psi(n)^{\dagger}\left(H(n-m) U(n, m \mid A)+\delta_{n m} M_{ \pm}(n \mid \phi)\right) \psi(m)
$$

where $H, U$ and $M_{ \pm}$are matrices defined as follows.

Firstly, the kinetic - or hopping - term, $H(n-m)$, invariant with respect to the lattice space group is represented by the Fourier integral,

$$
H(n-m)=\int_{B Z}\left(\frac{d p}{2 \pi}\right)^{4} e^{i p(n-m)} \gamma_{5}\left(i \gamma^{\mu} C_{\mu}(p)+B(p) T_{c}\right)
$$

where $p(n-m)=p_{\mu}(n-m)^{\mu}$ and the real functions, $C_{\mu}(p), B(p)$ are periodic under $p_{\mu} \rightarrow p_{\mu}+2 \pi$. They are invariant with respect to the lattice point group and are chosen such that, near $p=0$,

$$
C_{\mu}(p)=p_{\mu}+\ldots, \quad B(p)=r p^{2}+\ldots
$$

Their precise form elsewhere in the Brillouin zone is not important apart from the stipulation that they should not have a common zero anywhere except $p=0$.

The Dirac matrices are hermitian, satisfying

$$
\left\{\gamma^{\mu}, \gamma^{\nu}\right\}=2 g^{\mu \nu}, \quad\left\{\gamma^{\mu}, \gamma_{5}\right\}=0, \quad \gamma_{5}^{2}=1
$$


where $g_{\mu \nu}$ is the lattice metric.

The matrix $T_{c}$ commutes with the Dirac matrices and is diagonal with eigenvalues $+1(-1)$ corresponding to right (left) handed Weyl fermions. It also commutes with the gauge factor, given by the path ordered exponential,

$$
U(n, m \mid A)=P\left(\exp i \int_{m}^{n} A\right)
$$

In this formula the path is a straight line joining lattice sites $m$ and $n$,

$$
x^{\mu}(t)=t n^{\nu}+(1-t) m^{\mu}, \quad 0 \leq t \leq 1
$$

and $A$ is a slowly varying vector potential,

$$
A=d x^{\mu} A_{\mu}^{\alpha}(x) T_{\alpha}
$$

Finally, the "mass" term is given by the linear and gauge covariant expression,

$$
M_{ \pm}(n \mid \phi)=\gamma_{5}\left(\phi^{i}(n) T_{i} \pm \Lambda T_{c}\right)
$$

where the matrices $T_{i}$ incorporate Yukawa coupling parameters and specify the representation to which $\phi^{i}$ belongs,

$$
\left[T_{i}, T_{\alpha}\right]=i\left(t_{\alpha}\right)_{i}{ }^{j} T_{j}
$$

They commute with Dirac matrices but they are required to anticommute with the chirality matrix, $T_{c}$, since their role is to connect left handed $\left(T_{c}=-1\right)$ to right handed $\left(T_{c}=+1\right)$ fermions in order to generate mass,

$$
\left\{T_{i}, T_{c}\right\}=0
$$

The parameter, $\Lambda$, is a positive constant.

We shall treat the gauge field as a weak perturbation and the Higgs field as a constant, writing

$$
H_{ \pm}=H_{ \pm}^{0}+V
$$

where $H_{ \pm}^{0}$ and $V$ can be expressed as bilinears in the Fourier components,

$$
\psi(p)=\sum_{n} \psi(n) e^{-i p n}
$$

They are given as integrals over the Brillouin zone,

$$
\begin{aligned}
H_{ \pm}^{0} & =\int\left(\frac{d p}{2 \pi}\right)^{4} \psi(p)^{\dagger} H_{ \pm}(p) \psi(p) \\
V & =\int\left(\frac{d p_{1}}{2 \pi}\right)^{4}\left(\frac{d p_{2}}{2 \pi}\right)^{4} \psi\left(p_{1}\right)^{\dagger} V\left(p_{1}, p_{2}\right) \psi\left(p_{2}\right)
\end{aligned}
$$


where

$$
H_{ \pm}(p)=\gamma_{5}\left(i \gamma^{\mu} C_{\mu}(p)+\phi^{i} T_{i}+(B(p) \pm \Lambda) T_{c}\right)
$$

and

$$
\begin{aligned}
V\left(p_{1}, p_{2}\right)= & -\int\left(\frac{d k}{2 \pi}\right)^{4} A_{\mu}^{\alpha}(k)(2 \pi)^{4} \delta_{2 \pi}\left(-p_{1}+p_{2}+k\right) \int_{0}^{1} d t H\left(p_{1}-t k\right)^{, \mu} T_{\alpha} \\
& +\frac{1}{2} \int\left(\frac{d k_{1}}{2 \pi}\right)^{4}\left(\frac{d k_{2}}{2 \pi}\right)^{4} A_{\mu}^{\alpha}\left(k_{1}\right) A_{\nu}^{\beta}\left(k_{2}\right)(2 \pi)^{4} \delta_{2 \pi}\left(-p_{1}+p_{2}+k_{1}+k_{2}\right) \cdot \\
& \cdot \int_{0}^{1} d t_{1} d t_{2} H\left(p_{1}-t_{1} k_{1}-t_{2} k_{2}\right)^{, \mu \nu}\left(\theta\left(t_{1}-t_{2}\right) T_{\alpha} T_{\beta}+\theta\left(t_{2}-t_{1}\right) T_{\beta} T_{\alpha}\right) \\
& +\ldots
\end{aligned}
$$

where $H(p)^{, \mu}=\partial H(p) / \partial p_{\mu}$, etc., and $\delta_{2 \pi}$ is the periodic delta function defined by the lattice sum,

$$
(2 \pi)^{4} \delta_{2 \pi}(p)=\sum_{n} e^{i p n}
$$

The momentum integrals in (2.14) are over $\mathbb{R}^{4}$ but, since $A_{\mu}(x)$ is assumed to be slowly varying, its Fourier transform $A_{\mu}(k)$ is concentrated around $k=0$.

The fields $\psi(n)$ and $\psi(n)^{\dagger}$ act on a Fock space with the vacuum defined by $\psi(n)|0\rangle=0$. To apply the overlap prescription it is necessary to construct two distinct ground states, $|A, \phi+\rangle$ and $|A, \phi-\rangle$ corresponding to the respective Hamiltonians, $H_{+}$and $H_{-}$. For free fermions, $A=\phi=0$, these ground states $|+\rangle$ and $|-\rangle$ are obtained by filling the negative energy eigenstates of the respective 1-body Hamiltonians, (2.13). Modifications caused by non-vanishing $A$ and $\phi$ are then to be computed perturbatively. The aim is to compute the functional,

$$
e^{-\Gamma(A, \phi)}=\frac{\langle A, \phi+\mid A, \phi-\rangle}{\langle+\mid-\rangle}
$$

to be interpreted as the vacuum amplitude. The effective potential, defined by the ratio

$$
e^{-\Omega V(\phi)}=\frac{\langle 0, \phi+\mid 0, \phi-\rangle}{\langle+\mid-\rangle}
$$

where $\phi$ is constant and $\Omega$ is the volume (number of sites) of the lattice can be computed exactly. Our main concern, however, is the polarization tensor given by terms of second order [2] in $A$,

$$
\begin{aligned}
\Gamma_{(2)}(A, \phi)= & \frac{1}{2}\left\langle 0, \phi+\left|V G_{+}^{2} V\right| 0, \phi+\right\rangle+\frac{1}{2}\left\langle 0, \phi+\left|V G_{-}^{2} V\right| 0, \phi-\right\rangle- \\
& \frac{\left\langle 0, \phi+\left|\left(V G_{+}+G_{-} V++V G_{+} V G_{+}+V G_{+} G_{-} V+G_{-} V G_{-} V\right)\right| 0, \phi-\right\rangle}{\langle 0, \phi+\mid 0, \phi-\rangle}
\end{aligned}
$$


with constant $\phi$. The operators $G_{ \pm}$are defined by

$$
G_{ \pm}=-\frac{1-|0, \phi \pm\rangle\langle 0, \phi \pm|}{H_{ \pm}^{0}(\phi)}
$$

It is understood here that the free vacua are normalized zero energy eigenstates of $H_{ \pm}^{0}(\phi)$, and the Brillouin-Wigner phase convention [1], $\langle 0, \phi \pm \mid A, \phi \pm\rangle>0$, is used.

To evaluate the matrix elements in (2.17) it is useful to expand $\psi(p)$ in plane wave eigenstates of the 1-body free Hamiltonians (2.13),

$$
\psi(p)=\sum_{\sigma}\left(b_{ \pm}(p, \sigma) u_{ \pm}(p, \sigma)+d_{ \pm}^{\dagger}(p, \sigma) v_{ \pm}(p, \sigma)\right)
$$

where $b_{+}$and $d_{+}$annihilate the ground state $|0, \phi+\rangle$. The orthonormal eigenspinors $u_{+}$ and $v_{+}$satisfy

$$
\begin{aligned}
& H_{+}(p) u_{+}(p, \sigma)=\omega_{+}(p, \sigma) u_{+}(p, \sigma) \\
& H_{+}(p) v_{+}(p, \sigma)=-\omega_{+}(p, \sigma) v_{+}(p, \sigma)
\end{aligned}
$$

and likewise for the eigenspinors of $H_{-}(p)$. The energies $\omega_{ \pm}(p, \sigma)$ are positive, given by

$$
\omega_{ \pm}(p, \sigma)^{2}=g^{\mu \nu} C_{\mu}(p) C_{\nu}(p)+m_{\sigma}^{2}+(B(p) \pm \Lambda)^{2}
$$

where $m_{\sigma}^{2}$ is an eigenvalue of the mass matrix, $(\phi \cdot T)^{2}$. Details are given in Appendix A.

The sets $\left\{u_{+}, v_{+}\right\}$and $\left\{u_{-}, v_{-}\right\}$are both orthonormal and complete. They are related by a unitary transformation

$$
\begin{aligned}
& u_{-}=\cos \beta u_{+}-\sin \beta v_{+} \\
& v_{-}=\sin \beta u_{+}+\cos \beta v_{+}
\end{aligned}
$$

where the angle $\beta(p, \sigma)$ can be chosen to lie in the interval $(0, \pi / 2)$. The zeroes of $\cos \beta$ serve to determine the spectrum of fermion states in 4-dimensional spacetime. For example, the free fermion $2-$ point correlation function is given by

$$
\frac{\left\langle 0, \phi+\left|\psi(n) \psi(m)^{\dagger}\right| 0, \phi-\right\rangle}{\langle 0, \phi+\mid 0, \phi-\rangle}=\int\left(\frac{d p}{2 \pi}\right)^{4} G(p) e^{i p(n-m)}
$$

where

$$
G(p)=\sum_{\sigma} \frac{u_{+}(p, \sigma) u_{-}(p, \sigma)^{\dagger}}{\cos \beta(p, \sigma)}
$$

In Appendix $\mathrm{A}$ it is shown that in the region $p, \phi \ll \Lambda \ll 1, \beta$ approaches the value $\pi / 2$ and (2.22) reduces to the form [2]

$$
G(p) \simeq \Lambda \frac{1+\gamma_{5} T_{c}}{2}(i \not p+\phi \cdot T)^{-1} \gamma_{5}+\ldots
$$


corresponding to the propagation of light chiral fermions. One can show that there are no other light fermions if $C_{\mu}$ and $B$ have no common zeroes apart from $p=0$. This is how the overlap prescription avoids the doubling phenomenon.

Our main purpose is to study the fermionic contribution to vacuum polarization given by (2.17) using methods sketched in Appendix A, in particular the formula (A.12). A somewhat lengthy but straightforward calculation reduces this to the form

$$
\begin{aligned}
\Gamma_{(2)}(A, \phi)= & \frac{1}{2} \sum_{1,2} \frac{u_{+}(1)^{\dagger} V(1,2) v_{+}(2) v_{+}(2)^{\dagger} V(2,1) u_{+}(1)}{\left(\omega_{+}(1)+\omega_{+}(2)\right)^{2}} \\
+ & \frac{1}{2} \sum_{1,2} \frac{u_{-}(1)^{\dagger} V(1,2) v_{-}(2) v_{-}(2)^{\dagger} V(2,1) u_{-}(1)}{\left(\omega_{-}(1)+\omega_{-}(2)\right)^{2}} \\
& +\sum_{1}\left(\frac{v_{+}(1)^{\dagger} V(1,1) u_{+}(1)}{2 \omega_{+}(1)} \tan \beta_{1}-\frac{u_{-}(1)^{\dagger} V(1,1) v_{-}(1)}{2 \omega_{-}(1)} \tan \beta_{1}\right) \\
+ & \sum_{1,2} \frac{v_{+}(2)^{\dagger} V(2,1) u_{+}(1)}{\omega_{+}(1)+\omega_{+}(2)}\left(-\frac{u_{+}(1)^{\dagger} V(1,2) u_{+}(2)}{2 \omega_{+}(2)} \tan \beta_{2}+\right. \\
& \left.+\frac{v_{+}(1)^{\dagger} V(1,2) v_{+}(2)}{2 \omega_{+}(1)} \tan \beta_{1}+\frac{v_{+}(1)^{\dagger} V(1,2) u_{+}(2)}{2\left(\omega_{+}(1)+\omega_{+}(2)\right)} \tan \beta_{1} \tan \beta_{2}\right) \\
+ & \sum_{1,2} \frac{u_{-}(2)^{\dagger} V(2,1) v_{-}(1)}{\omega_{-}(1)+\omega_{-}(2)}\left(-\frac{v_{-}(1)^{\dagger} V(1,2) v_{-}(2)}{2 \omega_{-}(2)} \tan \beta_{2}+\right. \\
& \left.+\frac{u_{-}(1)^{\dagger} V(1,2) u_{-}(2)}{2 \omega_{-}(1)} \tan \beta_{1}+\frac{u_{-}(1)^{\dagger} V(1,2) v_{-}(2)}{2\left(\omega_{-}(1)+\omega_{-}(2)\right)} \tan \beta_{1} \tan \beta_{2}\right) \\
& -\sum_{1,2} \frac{u_{-}(1)^{\dagger} V(1,2) v_{-}(2) v_{+}(2)^{\dagger} V(2,1) u_{+}(1)}{\left(\omega_{+}(1)+\omega_{+}(2)\right)\left(\omega_{-}(1)+\omega_{-}(2)\right)} \frac{1}{\cos \beta_{1} \cos \beta_{2}}
\end{aligned}
$$

where the notation, $\sum_{1}$, indicates integration over $p_{1}$ and summation over $\sigma_{1}$. The vertex, $V(1,2)=V\left(p_{1}, p_{2}\right)$, is given by $(2.14)$ as a sum of first and second order pieces. These are to be inserted appropriately in (2.23) so as to obtain all terms of second order, i.e. the polarization tensor.

The daunting complexity of (2.23) is mainly due to unphysical structures associated with the extra degrees of freedom involved in the overlap prescription. We shall be concerned with its infrared behaviour in the next section and there it will be found that there are considerable simplifications.

Ward identities for the functional $\Gamma(A, \phi)$ are reviewed in Appendix B. There it is shown that, in second order,

$$
\begin{aligned}
& \partial_{\mu} \frac{\delta^{2} \Gamma}{\delta A_{\mu}^{\alpha}(x) \delta A_{\nu}^{\beta}(0)}-\phi^{T} t_{\alpha} \frac{\delta^{2} \Gamma}{\delta \phi(x) \delta A_{\nu}^{\beta}(0)}=0 \\
& \partial_{\mu} \frac{\delta^{2} \Gamma}{\delta A_{\mu}^{\alpha}(x) \delta \phi(0)}-\phi^{T} t_{\alpha} \frac{\delta^{2} \Gamma}{\delta \phi(x) \delta \phi(0)}=\delta_{4}(x) t_{\alpha} \frac{\delta \Gamma}{\delta \phi}
\end{aligned}
$$

where the derivatives are evaluated in the background $A_{\mu}=\partial_{\mu} \phi=0$. Thus, it is to be expected that the polarization tensor will include a longitudinal component if the Higgs 
field is non-vanishing. It represents a fermionic contribution to the effective kinetic term for the Higgs field.

\section{The continuum limit}

The vacuum polarization tensor is defined by the second order formula (2.23) which can be expressed in the form

$$
\Gamma_{(2)}(A, \phi)=\frac{1}{2} \int\left(\frac{d k}{2 \pi}\right)^{4} A_{\mu}^{\alpha}(-k) \Pi_{\alpha \beta}^{\mu \nu}(k, \phi) A_{\nu}^{\beta}(k)
$$

with

$$
\Pi_{\alpha \beta}^{\mu \nu}(k, \phi)=\int_{B Z}\left(\frac{d p}{2 \pi}\right)^{4} F_{\alpha \beta}^{\mu \nu}(p, k, \phi)
$$

Since $A_{\mu}(x)$ is assumed to be slowly varying, its Fourier transform $A_{\mu}(k)$ is concentrated around the point $k=0$. The Higgs background, $\phi$, is constant and, in order to identify the contributions of fermions with physically realistic masses we shall assume that it is a small quantity, of the same order as $k$.

At the point $k=\phi=0$ the integral (3.2) diverges. This is an infrared singularity, a threshold effect associated with the propagation of massless virtual fermions, and it dominates the low energy structure of the polarization tensor. Our task is to isolate this singularity and compute its contribution for small values of $k$ and $\phi$.

In the analytic continuation of (3.2) to complex values of $k$ other, unphysical, singularities are encountered. For example, if $\Lambda \ll 1$, there is a branch point at $k^{2}=-4 \Lambda^{2}$ that can be interpreted as the threshold for creation of a pair of fermions of mass, $\Lambda$. Such thresholds are artefacts of the overlap prescription and have no physical significance. The overlap method can be expected to give a physically trustworthy result only in the restricted region,

$$
k \sim \phi \ll \Lambda \ll 1
$$

The infrared singularity, being a threshold effect, must arise from the simultaneous vanishing of factors in the denominator of the integrand of (3.2). Since the momentum integral is 4-dimensional, it is necessary to have a zero of order 4 in the denominator in order to create an infrared divergence at $k=\phi=0$. Generically, there must be four factors, each contributing a simple zero. It is quite easy to identify the responsible terms and it will be seen that their zeroes occur at $p=0$ and nowhere else.

To identify the sources of vanishing denominators in (2.23) we need the explicit formulae for eigenspinors developed in Appendix A,

$$
u_{ \pm}(p, \sigma)=\frac{\omega_{ \pm}+H_{ \pm}(p)}{\sqrt{2 \omega_{ \pm}\left(\omega_{ \pm}+B \pm \Lambda\right)}} \chi(\sigma)
$$




$$
v_{ \pm}(p, \sigma)=\frac{\omega_{ \pm}-H_{ \pm}(p)}{\sqrt{2 \omega_{ \pm}\left(\omega_{ \pm}-B \mp \Lambda\right)}} \chi(\sigma)
$$

where $H_{ \pm}(p)$ are the 1-body Hamiltonians (2.13) and the positive energies $\omega_{ \pm}$are given by (2.20). Also,

$$
\begin{aligned}
\cos \beta & =v_{+}^{\dagger} v_{-} \\
& =\sqrt{\frac{\omega_{+}-B-\Lambda}{2 \omega_{+}} \frac{\omega_{-}-B+\Lambda}{2 \omega_{-}}}+\sqrt{\frac{\omega_{+}+B+\Lambda}{2 \omega_{+}} \frac{\omega_{-}+B-\Lambda}{2 \omega_{-}}}
\end{aligned}
$$

An inspection of the various terms in (2.23) shows that $\cos \beta$ must contribute a zero if we are to obtain a zero of order 4 . In order for $\cos \beta$ to vanish, both non-negative square roots in (3.5) must vanish. There are two possibilities, either

$$
\omega_{+}=B+\Lambda, \quad \omega_{-}=-B+\Lambda
$$

or else,

$$
\omega_{+}=-B-\Lambda, \quad \omega_{-}=B-\Lambda
$$

The second alternative can be excluded immediately because $\left(3.6^{\prime}\right)$ implies $\omega_{+}+\omega_{-}=-2 \Lambda$ which contradicts the positivity of $\omega_{ \pm}$. The first alternative is acceptable only if $\Lambda \pm B>0$, i.e. if $\Lambda^{2}>B^{2}$. On the other hand (3.6) implies $C_{\mu}=\phi=0$ as can be seen from the definition (2.20). Recall now, that it was part of the overlap prescription that $C_{\mu}$ and $B$ should have no common zeroes except for the origin, $p=0$. In particular, this means that $B$ should not vanish at any of the non-trivial zeroes of $C_{\mu}$. Hence, by choosing $\Lambda$ small enough we can ensure that the requirement $\Lambda \pm B>0$ is satisfied only at the origin where $B$ vanishes. We conclude that, if $\Lambda$ is small enough then the only zero of $\cos \beta$ is at $p=0$. At this point (3.6) also implies zeroes in the denominators of $u_{-}$and $v_{+}$. Hence, there is an infrared singularity and it resides in the terms,

$$
\begin{aligned}
\Gamma_{\text {sing }}(A, \phi) & =\sum_{1,2}\left[\frac{v_{+}(2)^{\dagger} V(2,1) u_{+}(1) v_{+}(1)^{\dagger} V(1,2) u_{+}(2)}{2\left(\omega_{+}(1)+\omega_{+}(2)\right)^{2}} \tan \beta_{1} \tan \beta_{2}\right. \\
& +\frac{u_{-}(2)^{\dagger} V(2,1) v_{-}(1) u_{-}(1)^{\dagger} V(1,2) v_{-}(2)}{2\left(\omega_{-}(1)+\omega_{-}(2)\right)^{2}} \tan \beta_{1} \tan \beta_{2} \\
& -\frac{v_{+}(2)^{\dagger} V(2,1) u_{+}(1) u_{-}(1)^{\dagger} V(1,2) v_{-}(2)}{\left(\omega_{+}(1)+\omega_{+}(2)\right)\left(\omega_{-}(1)+\omega_{-}(2)\right)} \cos \beta_{1} \cos \beta_{2}
\end{aligned}
$$

To identify the singularity we can expand around the point $p=k=\phi=0$ using

$$
\begin{aligned}
\omega_{ \pm} & =\Lambda+\frac{p^{2}+m_{\sigma}^{2}}{2 \Lambda} \pm r p^{2}+\ldots \\
\cos \beta & =\sqrt{\frac{p^{2}+m_{\sigma}^{2}}{\Lambda^{2}}}+\ldots \\
V(1,2) & =-i \gamma_{5} \gamma^{\mu} A_{\mu}^{\alpha}\left(p_{1}-p_{2}\right) T_{\alpha}+\ldots
\end{aligned}
$$


etc. Using the approximate expressions for the eigenspinors given in Appendix A one obtains the simple result,

$\Pi_{\alpha \beta}^{\mu \nu}(k, \phi)=-\int\left(\frac{d p}{2 \pi}\right)^{4} \operatorname{Tr}\left[\gamma^{\mu} T_{\alpha} \frac{1+\gamma_{5} T_{c}}{2} \frac{1}{i p_{1}+\phi \cdot T} \cdot \gamma^{\nu} T_{\beta} \frac{1+\gamma_{5} T_{c}}{2} \frac{}{i \not p_{2}+\phi \cdot T}+\ldots\right]$

where $p_{1}=p+\frac{k}{2}, \quad p_{2}=p-\frac{k}{2}$. Terms not shown here do not contribute to the infrared singularity.

To compute the singular terms contributed by (3.8) one can apply standard field theory methods. For example, using a Laplace transform representation for the vanishing denominator factors,

$$
\frac{1}{p_{1}^{2}+m_{1}^{2}} \frac{1}{p_{2}^{2}+m_{2}^{2}}=\int_{0}^{1} d x \int_{0}^{\infty} d t t e^{-t\left[x\left(p_{1}^{2}+m_{1}^{2}\right)+(1-x)\left(p_{2}^{2}+m_{2}^{2}\right)\right]}
$$

the integral can be expressed in the form

$$
\Pi_{\alpha \beta}^{\mu \nu}(k, \phi)=\int_{0}^{1} d x \int_{0}^{\infty} \frac{d t}{t} f_{\alpha \beta}^{\mu \nu}(t, x, k, \phi) e^{-t x(1-x) k^{2}}
$$

where

$$
\begin{aligned}
& f_{\alpha \beta}^{\mu \nu}(t, x, k, \phi)=-t^{2} \int\left(\frac{d p}{2 \pi}\right)^{4} e^{-t\left[x p_{1}^{2}+(1-x) p_{2}^{2}-x(1-x) k^{2}\right]} \\
& \cdot \operatorname{Tr}\left[\gamma^{\mu} T_{\alpha} \frac{1+\gamma_{5} T_{c}}{2}\left(-i p_{1}+\phi \cdot T\right) e^{-t x(\phi T)^{2}} \gamma^{\nu} T_{\beta} \frac{1+\gamma_{5} T_{c}}{2}\left(-i p_{2}+\phi \cdot T\right) e^{-t(1-x)(\phi T)^{2}}+\ldots\right]
\end{aligned}
$$

The behaviour at small $k^{2}$ of the integral (3.9) is dominated by the behaviour of $f$ at large $t$ and this can be determined from (3.10) by the method of steepest descents. Indeed it is clear that the factor $\exp \left(-t p^{2}\right)$ in the integrand of (3.10) serves to concentrate the support around $p=0$ as $t$ becomes large. This validates the expansion used in obtaining (3.8). The leading term in an asymptotic expansion in $1 / t$ is given by

$$
f_{\alpha \beta}^{\mu \nu} \simeq \frac{-2}{(4 \pi)^{2}}\left[\left\{-2 x(1-x)\left(k^{2} g^{\mu \nu}-k^{\nu} k^{\nu}\right)+g^{\mu \nu}\left(x(1-x) k^{2}+\frac{1}{t}\right)\right\} \rho_{\alpha \beta}+g^{\mu \nu} \sigma_{\alpha \beta}+\ldots\right]
$$

where $\rho_{\alpha \beta}$ and $\sigma_{\alpha \beta}$ are defined by the flavour traces,

$$
\begin{aligned}
\rho_{\alpha \beta} & =\operatorname{tr}\left(T_{\alpha} e^{-t x(\phi T)^{2}} T_{\beta} e^{-t(1-x)(\phi T)^{2}}\right) \\
\sigma_{\alpha \beta} & =\operatorname{tr}\left(T_{\alpha} \phi \cdot T e^{-t x(\phi \cdot T)^{2}} T_{\beta} \phi \cdot T e^{-t(1-x)(\phi \cdot T)^{2}}\right)
\end{aligned}
$$

On substituting (3.11) into (3.9) one obtains the asymptotic formula

$$
\begin{aligned}
\Pi_{\alpha \beta}^{\mu \nu}(k, \phi) \simeq & -\frac{2}{(4 \pi)^{2}} \int_{0}^{1} d x \int^{\infty} \frac{d t}{t}\left[-2 x(1-x) \rho_{\alpha \beta}(t, \phi)\left(k^{2} g^{\mu \nu}-k^{\mu} k^{\nu}\right)+\right. \\
& \left.+\left(\sigma_{\alpha \beta}(t, \phi)+\frac{\partial}{\partial t} \rho_{\alpha \beta}(t, \phi)\right) g^{\mu \nu}\right] e^{-t x(1-x) k^{2}}
\end{aligned}
$$


valid for $k, \phi \rightarrow 0$. The integral over $t$ can be written as a linear combination of terms like

$$
\int^{\infty} \frac{d t}{t} e^{-t M^{2}}=-\ell n M^{2}+c_{0}+c_{1} M^{2}+\ldots
$$

for $M^{2} \rightarrow 0$. The leading term is well defined but the subleading ones are sensitive to what happens at finite values of $t$ where the expression (3.11) is not adequate.

In Sec. 4 a specific example drawn from the standard model will be considered in more detail. To conclude the present discussion we remark only that the flavour traces (3.12) take the form

$$
\rho_{\alpha \beta}=\sum \rho_{\alpha \beta}^{i} e^{-t m_{i}^{2}}, \quad \sigma_{\alpha \beta}=\sum \sigma_{\alpha \beta}^{i} e^{-t m_{i}^{2}}
$$

where $m_{i}$ is a fermion mass. Substituting these expressions into (3.13) and applying (3.14) one obtains

$$
\begin{aligned}
\Pi_{\alpha \beta}^{\mu \nu}(k, \phi) \simeq & -\frac{2}{(4 \pi)^{2}} \int_{0}^{1} d x\left[2 x(1-x) \rho_{\alpha \beta}^{i}\left(k^{2} g^{\mu \nu}-k^{\mu} k^{\nu}\right)-\right. \\
& \left.-\left(\sigma_{\alpha \beta}^{i}-m_{i}^{2} \rho_{\alpha \beta}^{i}\right) g^{\mu \nu}\right] \ln \left(m_{i}^{2}+x(1-x) k^{2}\right)
\end{aligned}
$$

valid for $m, k \ll \Lambda \ll 1$.

\section{Standard model}

To study the application of the overlap prescription to the standard model we consider the continuum limit of Sec.3 for the gauge group $S U(2) \times U(1)$ with a single Higgs doublet and four chiral fermions, a left handed doublet and two right handed singlets,

$$
\phi=\left(\begin{array}{l}
u_{L} \\
d_{L} \\
u_{R} \\
d_{R}
\end{array}\right)
$$

The generators of $S U(2) \times U(1)$ are represented by the $4 \times 4$ matrices,

$$
\begin{aligned}
& T_{a}=\left(\begin{array}{ccc}
\frac{1}{2} \tau_{a} & & \\
& 0 & \\
& & 0
\end{array}\right), \quad a=1,2,3 \\
& T_{Y}=\left(\begin{array}{lll}
Y_{L} & & \\
& Y_{u} & \\
& & Y_{d}
\end{array}\right)
\end{aligned}
$$

where the hypercharge assignments are given by

$$
\left(Y_{L}, Y_{u}, Y_{d}\right)=\left\{\begin{array}{rr}
(1 / 3,4 / 3,-2 / 3), & \text { quarks } \\
(-1,0,-2), & \text { leptons }
\end{array}\right.
$$


(The Higgs doublet carries $Y_{\phi}=1$.) The chirality matrix is

$$
T_{c}=\left(\begin{array}{ccc}
-\mathbb{1} & & \\
& 1 & \\
& & 1
\end{array}\right)
$$

and the Higgs-Yukawa matrix is

$$
\phi \cdot T=\left(\begin{array}{ccc}
0 & f_{u} \bar{\phi} & f_{d} \phi \\
f_{u} \bar{\phi}^{\dagger} & 0 & 0 \\
f_{d} \phi^{\dagger} & 0 & 0
\end{array}\right)
$$

where $f_{u}$ and $f_{d}$ are real Yukawa coupling constants and

$$
\phi=\left(\begin{array}{c}
\phi_{1} \\
\phi_{2}
\end{array}\right), \quad \bar{\phi}=\left(\begin{array}{c}
\phi_{2}^{*} \\
-\phi_{1}^{*}
\end{array}\right)
$$

The matrices $T_{a}, T_{c}$ and $\phi \cdot T$ are all hermitian.

It is a simple exercise to compute the flavour traces $\rho_{\alpha \beta}$ and $\sigma_{\alpha \beta}$ defined by (3.12). One finds

$$
\begin{aligned}
\rho_{a b}= & \frac{1}{4} \delta_{a b}\left(e^{-t\left(x m_{u}^{2}+(1-x) m_{d}^{2}\right)}+e^{-t\left(x m_{d}^{2}+(1-x) m_{u}^{2}\right)}\right) \\
& +\frac{1}{4} \frac{\phi^{\dagger} \sigma_{a} \phi \phi^{\dagger} \sigma_{b} \phi}{\left(\phi^{\dagger} \phi\right)^{2}}\left[e^{-t m_{u}^{2}}+e^{-t m_{d}^{2}}\right. \\
& \left.-e^{-t\left(x m_{u}^{2}+(1-x) m_{d}^{2}\right)}-e^{-t\left(x m_{d}^{2}+(1-x) m_{u}^{2}\right)}\right] \\
\rho_{a Y}= & \frac{Y_{L}}{2} \frac{\phi^{\dagger} \tau_{a} \phi}{\phi^{\dagger} \phi}\left(-e^{-t m_{u}^{2}}+e^{\left.-t m_{d}^{2}\right)}\right. \\
\rho_{Y Y}= & \left(Y_{L}^{2}+Y_{u}^{2}\right) e^{-t m_{u}^{2}}+\left(Y_{L}^{2}+Y_{d}^{2}\right) e^{-t m_{d}^{2}} \\
\sigma_{a b}= & 0 \\
\sigma_{a Y}= & \frac{1}{2} \frac{\phi^{\dagger} \tau_{a} \phi}{\phi^{\dagger} \phi}\left(-Y_{u} m_{u}^{2} e^{-t m_{u}^{2}}+Y_{d} m_{d}^{2} e^{-t m_{d}^{2}}\right) \\
\sigma_{Y Y}= & 2 Y_{L} Y_{u} m_{u}^{2} e^{-t m_{u}^{2}}+2 Y_{L} Y_{d} m_{d}^{2} e^{-t m_{d}^{2}}
\end{aligned}
$$

where $m_{u}^{2}=f_{u}^{2} \phi^{\dagger} \phi$ and $m_{d}^{2}=f_{d}^{2} \phi^{\dagger} \phi$. In writing $\rho_{a b}$ we dropped a term which is antisymmetric with respect to $x \leftrightarrow 1-x$. The coefficients $\rho_{\alpha \beta}^{i}$ and $\sigma_{\alpha \beta}^{i}$ of (3.15) are thereby determined and can be substituted into (3.16). We list the transverse and longitudinal components separately, writing

$$
\Pi_{\alpha \beta}^{\mu \nu}(k, \phi)=\left(k^{2} g^{\mu \nu}-k^{\mu} k^{\nu}\right) \Pi_{\alpha \beta}+g^{\mu \nu} H_{\alpha \beta}
$$

The results are

$$
\Pi_{a b}=\frac{1}{16 \pi^{2}} \int_{0}^{1} d x x(1-x)\left[-2 \delta_{a b} \ln \left(x m_{u}^{2}+(1-x) m_{d}^{2}+x(1-x) k^{2}\right)\right.
$$




$$
\begin{aligned}
& +\frac{\phi^{\dagger} \tau_{a} \phi \phi^{\dagger} \tau_{b} \phi}{\left(\phi^{\dagger} \phi\right)^{2}}\left\{-\ln \left(m_{u}^{2}+x(1-x) k^{2}\right)-\ell n\left(m_{d}^{2}+x(1-x) k^{2}\right)\right. \\
& \left.+2 \ln \left(x m_{u}^{2}+(1-x) m_{d}^{2}-x(1-x) k^{2}\right\}\right] \\
& \Pi_{a Y}=\frac{Y_{L}}{8 \pi^{2}} \frac{\phi^{\dagger} \tau_{a} \phi}{\phi^{\dagger} \phi} \int_{0}^{1} d x x(1-x) \ln \left(\frac{m_{u}^{2}+x(1-x) k^{2}}{m_{d}^{2}+x(1-x) k^{2}}\right) \\
& \Pi_{Y Y}=-\frac{1}{4 \pi^{2}} \int_{0}^{1} d x x(1-x)\left[\left(Y_{L}^{2}+Y_{u}^{2}\right) \ln \left(m_{u}^{2}+x(1-x) k^{2}\right)\right. \\
& \left.+\left(Y_{L}^{2}-Y_{d}^{2}\right) \ln \left(m_{d}^{2}+x(1-x) k^{2}\right)\right] \\
& H_{a b}=\frac{1}{16 \pi^{2}} \int_{0}^{1} d x\left[-\delta_{a b}\left(x m_{u}^{2}+(1-x) m_{d}^{2}\right) \ln \left(x m_{u}^{2}+(1-x) m_{d}^{2}+x(1-x) k^{2}\right)\right. \\
& -\frac{1}{2} \frac{\phi^{\dagger} \tau_{a} \phi^{\dagger} \tau_{b} \phi}{\left(\phi^{\dagger} \phi\right)^{2}}\left\{m_{u}^{2} \ln \left(m_{u}^{2}+x(1-x) k^{2}\right)+m_{d}^{2} \ln \left(m_{d}^{2}+x(1-x) k^{2}\right)\right. \\
& \left.\left.-2\left(x m_{u}^{2}+(1-x) m_{d}^{2}\right) \ln \left(x m_{u}^{2}+(1-x) m_{d}^{2}+x(1-x) k^{2}\right)\right\}\right] \\
& H_{a Y}=\frac{1}{16 \pi^{2}} \frac{\phi^{\dagger} \tau_{a} \phi}{\phi^{\dagger} \phi} \int_{0}^{1} d x\left[\left(Y_{L}-Y_{u}\right) m_{u}^{2} \ln \left(m_{u}^{2}+x(1-x) k^{2}\right)-\right. \\
& \left.-\left(Y_{L}-Y_{d}\right) m_{d}^{2} \ln \left(m_{d}^{2}+x(1-x) k^{2}\right)\right] \\
& H_{Y Y}=-\frac{1}{8 \pi^{2}} \int_{0}^{1} d x\left[\left(Y_{L}-Y_{u}\right)^{2} m_{u}^{2} \ln \left(m_{u}^{2}+x(1-x) k^{2}\right)+\right. \\
& \left.+\left(Y_{L}-Y_{d}\right)^{2} m_{d}^{2} \ln \left(m_{u}^{2}+x(1-x) k^{2}\right)\right]
\end{aligned}
$$

These expressions comprise the leading, or infrared singular, part of the unrenormalized polarization tensor. The first subleading terms, corresponding to the parameters, $c_{0}$, in (3.14) would take the form of a second order polynomial in $k$. Because gauge invariance is guaranteed, this polynomial whose coefficients must depend on $\Lambda$ as well as details of the functions $C_{\mu}$ and $B$, can be absorbed in a counterterm. Higher order effects, involving $c_{1}, \ldots$ become vanishingly small in the infrared limit and can be discarded. Therefore, the only remaining dependence on the lattice regularization is in the system of units we have employed. This also can be absorbed in a counterterm.

Define the renormalized polarization tensor by introducing a reference mass, $\mu$, and using it to scale the arguments of all the logarithms in the expressions (4.9), i.e. make the replacements, $k^{2} \rightarrow k^{2} / \mu^{2}, m^{2} \rightarrow m^{2} / \mu^{2}$. This generates a counterterm proportional 
to $\ln \mu^{2}$,

$$
\Gamma=\Gamma^{R}+\Delta \Gamma
$$

where the counterterm is given by the gauge invariant local expression

$$
\begin{array}{r}
\Delta \Gamma=-\ell n \mu^{2} \int d^{4} x\left[\frac{1}{48 \pi^{2}} \frac{1}{4}\left(F_{\mu \nu}^{a}\right)^{2}+\frac{2 Y_{L}^{2}+Y_{u}^{2}+Y_{d}^{2}}{24 \pi^{2}} \frac{1}{4}\left(F_{\mu \nu}^{Y}\right)^{2}+\right. \\
\left.+\frac{f_{u}^{2}+f_{d}^{2}}{16 \pi^{2}} \nabla_{\mu} \phi^{\dagger} \nabla_{\mu} \phi\right]
\end{array}
$$

It can be combined with corresponding terms in the classical action to define running coupling constants

$$
\begin{aligned}
& \frac{1}{g_{I}^{2}}=\frac{1}{g_{0 I}^{2}}-\frac{1}{48 \pi^{2}} \ln \mu^{2} \\
& \frac{1}{g_{Y}^{2}}=\frac{1}{g_{0 Y}^{2}}-\frac{2 Y_{L}^{2}+Y_{u}^{2}+Y_{d}^{2}}{24 \pi^{2}} \ln \mu^{2}
\end{aligned}
$$

and the wave function renormalization

$$
Z_{\phi}=1-\frac{f_{u}^{2}+f_{d}^{2}}{16 \pi^{2}} \ln \mu^{2}
$$

The fermionic contributions to the Callan-Symanzik functions are therefore given by

$$
\begin{aligned}
\mu \frac{\partial g_{I}}{\partial \mu} & =\frac{1}{48 \pi^{2}} g_{I}^{3} \\
\mu \frac{\partial g_{Y}}{\partial \mu} & =\frac{2 Y_{L}^{2}+Y_{u}^{2}+Y_{d}^{2}}{24 \pi^{2}} g_{Y}^{3} \\
& = \begin{cases}\frac{22 / 9}{24 \pi^{2}} g_{Y}^{3}, & \text { quarks } \\
\frac{6}{24 \pi^{2}} g_{Y}^{3}, & \text { leptons }\end{cases} \\
\mu \frac{\partial \ln Z_{\phi}^{-1}}{\partial \mu} & =\frac{f_{u}^{2}+f_{d}^{2}}{8 \pi^{2}}
\end{aligned}
$$

\section{Summary}

Our purpose in this work was to show that a lattice formulation of gauge theories with chiral fermions is satisfactory in the lowest order of perturbation theory. We have computed the vacuum polarization due to fermions and verified that it reduces in the low energy approximation - or, equivalently, when the lattice spacing goes to zero - to the expected form. In obtaining this result it was crucial that the free fermion spectrum contains no light doublers; a feature that can be ensured by appropriate choice of the free Hamiltonians.

The possibility to choose Hamiltonians such that light fermions are not doubled seems to be the real strength of the overlap prescription. It should be emphasized that this 
has nothing to do with the Higgs mechanism. We introduced a Higgs multiplet into the system only in order to make it phenomenologically more interesting. This field played no role in solving the doubling problem.

Our approach in this work has been to emphasize the infrared nature of the problem by scaling with respect to the ultraviolet cutoff, i.e. working in lattice spacing units. Integrals that are not infrared dominated can be set aside and absorbed in the usual counterterms. On the other hand, integrals that are infrared dominated become singular when the masses and external momenta are scaled to zero, and these singular contributions can be computed without regard to short distance structure. They necessarily coincide with what is usually called "the finite part" in Feynman graph calculations. As it happens, the amplitude treated here is gauge invariant and hence so are the counterterms. But this is not important. Evaluation of counterterms necessarily involves integrating over the entire Brillouin zone and this would be a difficult task.

Of course, this is only a second order calculation. In previous work it was shown that the expected chiral anomalies begin to emerge in the next order, and that is also satisfactory. However, not much is known about the general structure of the overlap amplitude, apart from several numerical studies [1], [16] which support the viability of the formalism.

\section{Acknowledgments}

We are appreciative of useful conversations with H. Arfaei and with H. Schlereth who brought Ref.[6] to our attention. Fruitful correspondence with H. Neuberger is also acknowledged. 


\section{Appendix A - Free fermion states}

To compute overlaps and matrix elements for free fermions ( $A=0, \phi=$ constant) it is necessary first to diagonalize the Hamiltonians $H_{ \pm}^{0}$ and construct their respective ground states. To this end consider the 1-body operators given by (2.9a)

$$
H_{ \pm}(p)=\gamma_{5}\left(i \gamma^{\mu} C_{\mu}(p)+\phi^{i} T_{i}+(B(p) \pm \Lambda) T_{c}\right)
$$

The square of this matrix,

$$
H_{ \pm}(p)^{2}=g^{\mu \nu} C_{\mu} C_{\nu}+(\phi \cdot T)^{2}+(B \pm \Lambda)^{2}
$$

is free of Dirac matrices and we can diagonalize it by choosing momentum independent orthonormal spinors $\chi(\sigma)$ such that

$$
(\phi \cdot T)^{2} \chi(\sigma)=m_{\sigma}^{2} \chi(\sigma)
$$

It is convenient also to impose the condition

$$
\gamma_{5} T_{c} \chi(\sigma)=\chi(\sigma)
$$

Orthonormal eigenspinors of (A.1) can then be written,

$$
\begin{aligned}
& u_{ \pm}(p, \sigma)=\frac{\omega_{ \pm}+H_{ \pm}(p)}{\sqrt{2 \omega_{ \pm}\left(\omega_{ \pm}+B \pm \Lambda\right)}} \chi(\sigma) \\
& v_{ \pm}(p, \sigma)=\frac{\omega_{ \pm}-H_{ \pm}(p)}{\sqrt{2 \omega_{ \pm}\left(\omega_{ \pm}-B \mp \Lambda\right)}} \chi(\sigma)
\end{aligned}
$$

where the eigenvalues are given by

$$
\omega_{ \pm}(p, \sigma)=\sqrt{g^{\mu \nu} C_{\mu} C_{\nu}+m_{\sigma}^{2}+(B \pm \Lambda)^{2}}
$$

These numbers are chosen to be positive so that $u_{ \pm}$and $v_{ \pm}$are, respectively, positive and negative energy eigenspinors of $H_{ \pm}(p)$. The sets $\left\{u_{+}, v_{+}\right\}$and $\left\{u_{-}, v_{-}\right\}$are both orthonormal and complete. They are related by a unitary transformation,

$$
\begin{aligned}
& u_{-}=\cos \beta u_{+}-\sin \beta v_{+} \\
& v_{-}=\sin \beta u_{+}+\cos \beta v_{+}
\end{aligned}
$$

where the angle $\beta=\beta(p, \sigma)$ lies in the range $(-\pi / 2, \pi / 2)$,

$$
\begin{aligned}
& \cos \beta=\sqrt{\frac{\omega_{+}-B-\Lambda}{2 \omega_{+}} \frac{\omega_{-}-B+\Lambda}{2 \omega_{-}}}+\sqrt{\frac{\omega_{+}+B+\Lambda}{2 \omega_{+}} \frac{\omega_{-}+B-\Lambda}{2 \omega_{-}}} \\
& \sin \beta=\sqrt{\frac{\omega_{+}+B+\Lambda}{2 \omega_{+}} \frac{\omega_{-}-B+\Lambda}{2 \omega_{-}}}-\sqrt{\frac{\omega_{+}-B-\Lambda}{2 \omega_{+}} \frac{\omega_{-}+B-\Lambda}{2 \omega_{-}}}
\end{aligned}
$$


Creation and annihilation operators for 1-fermion states are defined by the plane wave expansions,

$$
\psi(n)=\int\left(\frac{d p}{2 \pi}\right)^{4} e^{i p n} \sum_{\sigma}\left(b_{ \pm}(p, \sigma) u_{ \pm}(p, \sigma)+d_{ \pm}^{\dagger}(p, \sigma) v_{ \pm}(p, \sigma)\right)
$$

They are related by a Bogoliubov transformation,

$$
\begin{aligned}
& b_{-}=\cos \beta b_{+}-\sin \beta d_{+}^{\dagger} \\
& d_{-}^{\dagger}=\sin \beta b_{+}+\cos \beta d_{+}^{\dagger}
\end{aligned}
$$

The Dirac vacua are defined by filling the negative energy states,

$$
| \pm\rangle=\prod_{k, \sigma} d_{ \pm}(k, \sigma)|0\rangle
$$

and their overlap is easily obtained,

$$
\langle+\mid-\rangle=\prod_{k, \sigma} \cos \beta(k, \sigma)
$$

It is now straightforward to construct correlation functions. For example, the 2-point function,

$$
\begin{aligned}
\frac{\left\langle+\left|\psi(n) \psi^{\dagger}(m)\right|-\right\rangle}{\langle+\mid-\rangle} & =\int\left(\frac{d p}{2 \pi}\right)^{4}\left(\frac{d p^{\prime}}{2 \pi}\right)^{4} \sum_{\sigma \sigma^{\prime}} u_{+}(p, \sigma) \frac{\left\langle+\left|b_{+}(p, \sigma) b_{-}^{\dagger}\left(p^{\prime}, \sigma^{\prime}\right)\right|-\right\rangle}{\langle+\mid-\rangle} u_{-}^{\dagger}\left(p^{\prime}, \sigma^{\prime}\right) \\
& =\int_{B Z}\left(\frac{d p}{2 \pi}\right)^{4} \sum_{\sigma} \frac{u_{+}(p, \sigma) u_{-}^{\dagger}(p, \sigma)}{\cos \beta(p, \sigma)}
\end{aligned}
$$

It is instructive to examine the long distance behaviour of this correlation function. For $p \sim \phi \ll \Lambda \ll 1$, the energies (A.5) are approximated by

$$
\omega_{ \pm}(p, \sigma) \simeq \Lambda+\frac{p^{2}+m_{\sigma}^{2}}{2 \Lambda} \pm r p^{2}+\ldots
$$

The eigenspinors (A.4) reduce to the form

$$
u_{+}(p, \sigma) \simeq \chi(\sigma), \quad u_{-}(p, \sigma) \simeq \gamma_{5} \frac{i \not p+\phi \cdot T}{\sqrt{p^{2}+m_{\sigma}^{2}}} \chi
$$

and

$$
\cos \beta(p, \sigma) \simeq \sqrt{\frac{p^{2}+m_{\sigma}^{2}}{\Lambda^{2}}}+\ldots
$$

Hence, in this neighbourhood,

$$
\sum_{\sigma} \frac{u_{+}(p, \sigma) u_{-}^{\dagger}(p, \sigma)}{\cos \beta(p, \sigma)} \simeq \Lambda \frac{1+\gamma_{5} T_{c}}{2} \frac{1}{i \not p+\phi \cdot T} \gamma_{5}+\ldots
$$


and the correlator (A.10) approximates - apart from the factors $\Lambda$ and $\gamma_{5}$, which can be removed by a suitable redefinition of the fields, - a chiral Euclidean propagator. The pole of the propagator is due to a zero in $\cos \beta$, i.e. a zero in the overlap (A.9).

Using the expansions $\left(\mathrm{A} .7^{\prime}\right)$ one can express the perturbation $V$ in terms of creation and annihilation operators,

$$
\begin{aligned}
V= & \sum_{12}\left[b(1)^{\dagger} u(1)^{\dagger} V(1,2) u(2) b(2)+b(1)^{\dagger} u(1)^{\dagger} V(1,2) v(2) d(2)^{\dagger}\right. \\
& \left.+d(1) v(1)^{\dagger} V(1,2) u(2) b(2)+d(1) v(1)^{\dagger} V(1,2) v(2) d(2)^{\dagger}\right]
\end{aligned}
$$

where the symbol $\sum_{12}$ denotes integration over $p_{1}, p_{2}$ and summation over $\sigma_{1}, \sigma_{2}$. The creation and annihilation operators $b(1)^{\dagger}, d(1)$ together with their associated eigenspinors $u(1)^{\dagger}, v(1)^{\dagger}$ may be of the + or - type. Likewise for $b(2), d(2)^{\dagger}$, etc. The operator $V$ either creates pairs or scatter. In second order one finds either 2 - or 4-particle intermediate states. The only unusual feature of the overlap computations is the appearance of matrix elements like

$$
\begin{aligned}
\langle 1, \overline{2}+\mid-\rangle & =\left\langle+\left|d_{+}(2) b_{+}(1)\right|-\right\rangle \\
& =\left\langle+\left|d_{+}(2) d_{-}(1)^{\dagger}\right|-\right\rangle \sin \beta(1) \\
& =\delta(1,2)\langle+\mid-\rangle \tan \beta(1)
\end{aligned}
$$

where $\delta(1,2)$ stands for $(2 \pi)^{4} \delta_{2 \pi}\left(p_{1}-p_{2}\right) \delta_{\sigma_{1} \sigma_{2}}$. Here we have used (A.8) to express $b_{+}(1)$ in terms of $b_{-}(1)$ and $d_{-}(1)^{\dagger}$. The ground state $|-\rangle$ is annihilated by $b_{-}(1)$. In passing to the next line we have used the formulae $\left(\right.$ A. $\left.8^{\prime}\right)$, (A.9) together with the Fock condition $d_{ \pm}^{\dagger}|0\rangle=0$. In commuting $d_{-}(1)^{\dagger}$ to the right it creates a gap at $k_{1}, \sigma_{1}$ in the product over $d_{-}$that defines the ground state $|-\rangle$. Likewise, in commuting $d_{+}(2)$ to the left it creates a gap at $k_{2}, \sigma_{2}$ in the product over $D_{+}^{\dagger}$ that defines $\langle+|$. The resulting states are orthogonal unless $1=2$ in which case their overlap equals $\langle+\mid-\rangle / \cos \beta(1)$. Quite generally, any overlap matrix element can be reduced with the help of $\left(\mathrm{A} .^{*}\right)$ to a linear combination of terms like

$$
\begin{aligned}
& \left\langle+\left|d_{+}\left(i_{1}\right) \ldots d_{+}\left(i_{n}\right) d_{-}^{\dagger}\left(j_{n}\right) \ldots d_{-}^{\dagger}\left(j_{1}\right)\right|-\right\rangle= \\
& \langle+\mid-\rangle\left(\cos \beta\left(i_{1}\right) \ldots \cos \beta\left(i_{n}\right)\right)^{-1} \sum_{\pi} \varepsilon_{\pi} \delta_{i_{1}, j_{\pi_{1}}} \ldots \delta_{i_{n} j_{\pi_{n}}}
\end{aligned}
$$

where $\pi_{1}, \ldots, \pi_{n}$ is a permutation of $1, \ldots, n$. 


\section{Appendix B - Ward identities}

Gauge transformations of the fermion fields can be implemented by unitary operators,

$$
\psi(n) \rightarrow e^{i \theta(n)} \psi(n)=e^{-i F_{\theta}} \psi(n) e^{i F_{\theta}}
$$

where $\theta(n)$ is an hermitian matrix and

$$
F_{\theta}=\sum_{n} \psi(n)^{\dagger} \theta(n) \psi(n)
$$

The Hamiltonians (2.1) are covariant in the sense

$$
e^{i F_{\theta}} H_{ \pm}(A, \phi) e^{-i F_{\theta}}=H_{ \pm}\left(A^{\theta}, \phi^{\theta}\right)
$$

where the transformed background fields $A^{\theta}$ and $\phi^{\theta}$ are defined by

$$
\begin{aligned}
e^{i \theta(n)} \phi(n) \cdot T e^{-i \theta(n)} & =\phi^{\theta}(n) \cdot T \\
e^{i \theta(n)} U(n, m \mid A) e^{-i \theta(m)} & =U\left(n, m \mid A^{\theta}\right)
\end{aligned}
$$

Since the functional $U(n, m \mid A)$ is defined by the path-ordered exponential (2.4) it follows that $A^{\theta}(x)$ is given by

$$
A_{\mu}^{\theta}(x)=e^{i \theta(x)}\left(A_{\mu}(x)+i \partial_{\mu}\right) e^{-i \theta(x)}
$$

where $\theta(x)$ is a smooth function that interpolates the lattice values, $\theta(n)$.

With Hamiltonians transforming according to (B.3) it follows that the corresponding non-degenerate ground states must transform such that

$$
e^{i F_{\theta}}|A, \phi \pm\rangle=\left|A^{\theta}, \phi^{\theta} \pm\right\rangle e^{i \Phi_{ \pm}(\theta, A, \phi)}
$$

where the angles $\Phi_{ \pm}$are real. The effective action (2.10) therefore satisfies the identity

$$
\Gamma\left(A^{\theta}, \phi^{\theta}\right)=\Gamma(A, \phi)+i \Phi_{+}(\theta, A, \phi)-i \Phi_{-}(\theta, A, \phi)
$$

Its real part is gauge invariant, but its imaginary part is not, unless $\Phi_{+}=\Phi_{-}$. These angles can be computed perturbatively. To first order in $\theta$, for example,

$$
\begin{aligned}
\Phi_{+} & =\operatorname{Re} \frac{\left\langle+\left|F_{\theta}\right| A, \phi+\right\rangle}{\langle+\mid A, \phi+\rangle} \\
& =\operatorname{Re}\left\langle+\left|F_{\theta}\left(1+G_{+} V+G_{+} V G_{+} V+\ldots\right)\right|+\right\rangle
\end{aligned}
$$

where the Brillouin-Wigner phase convention, $\langle+\mid A, \phi+\rangle>0$, is assumed. The difference, $\Phi_{+}-\Phi_{-}$, is a pseudoscalar with respect to space reflections and it can be shown to vanish up to first order in $A_{\mu}$ and $\partial_{\mu} \phi$ in dimensions $\geq 4$. In second order it can be non-vanishing, thereby reflecting the usual anomalous breakdown of gauge symmetry associated with chiral fermions. 
The formula (B.7) comprises the Ward identities for the fermion vacuum amplitude. For infinitesimal $\theta$ we have, from (B.4) and (B.5)

$$
\begin{aligned}
\delta A_{\mu}(x) & =\partial_{\mu} \theta(x)-i\left[A_{\mu}(x), \theta(x)\right] \\
& =\nabla_{\mu} \theta(x) \\
\delta \phi(x) \cdot T & =i[\theta(x), \phi(x) \cdot T]
\end{aligned}
$$

where $\phi(x)$ is a multiplet of smooth fields that interpolates the lattice values $\phi(n)$. For writing Ward identities it is convenient to choose a hermitian basis with real components, $\phi^{i}(x)$,

$$
\begin{aligned}
\phi \cdot T & =\phi^{i} T_{i} \\
{\left[T_{j}, T_{\alpha}\right] } & =i\left(t_{\alpha}\right)_{j}{ }^{i} T_{i}
\end{aligned}
$$

so that the components transform according to

$$
\delta \phi^{i}(x)=-\theta^{\alpha}(x) \phi^{j}(x)\left(t_{\alpha}\right)_{j}^{i}
$$

With this notation the identity (B.7) implies

$$
\nabla_{\mu} \frac{\delta \Gamma}{\delta A_{\mu}^{\alpha}}+\phi^{j}\left(t_{\alpha}\right)_{j}{ }^{i} \frac{\delta \Gamma}{\delta \phi^{i}}=-i \frac{\delta}{\delta \theta^{\alpha}}\left(\Phi_{+}-\Phi_{-}\right)
$$

and, in particular, for the second derivates evaluated at $A_{\mu}=\partial_{\mu} \phi=0$,

$$
\begin{aligned}
& \partial_{\mu} \frac{\delta^{2} \Gamma}{\delta A_{\mu}^{\alpha}(x) \delta A_{\nu}^{\beta}(0)}+\phi^{T} t_{\alpha} \frac{\delta^{2} \Gamma}{\delta \phi(x) \delta A_{\nu}^{\beta}(0)}=0 \\
& \partial_{\mu} \frac{\delta^{2} \Gamma}{\delta A_{\mu}^{\alpha}(x) \delta \phi(0)}+\phi^{T} t_{\alpha} \frac{\delta^{2} \Gamma}{\delta \phi(x) \delta \phi(0)}=-\delta_{4}(x) t_{\alpha} \frac{\delta \Gamma}{\delta \phi}
\end{aligned}
$$

since $\Phi_{+}=\Phi_{-}$does not contribute here. The vacuum polarization tensor is not transverse unless $\phi=0$. Its longitudinal part is related to the Higgs field kinetic term. To see what is implied by this relation, consider the leading terms in a continuum approximation,

$$
\Gamma(A, \phi) \simeq \int d^{4} x\left[\frac{Z_{3}}{4}\left(F_{\mu \nu}^{\alpha}\right)^{2}+\frac{Z_{2}}{2}\left(\nabla_{\mu} \phi^{i}\right)^{2}+\ldots\right]
$$

where $Z_{2}$ and $Z_{3}$ are invariant functions of $\phi(x)$. For this simplified functional we have

$$
\begin{aligned}
\frac{\delta^{2} \Gamma}{\delta A_{\mu}^{\alpha}(x) \delta A_{\nu}^{\beta}(0)}= & Z_{3} \delta_{\alpha \beta}\left(\delta_{\mu \nu} \partial^{2}-\partial_{\mu} \partial_{\nu}\right) \delta_{4}(x) \\
& -Z_{2} \phi^{T} t_{\alpha} t_{\beta} \phi \delta_{\mu \nu} \delta_{4}(x) \\
\frac{\delta^{2} \Gamma}{\delta \phi(x) \delta A_{\nu}^{\beta}(0)}= & Z_{2} t_{\beta} \phi \partial_{\nu} \delta_{4}(x)
\end{aligned}
$$

which clearly satisfies (B.13a). 


\section{References}

[1] R. Narayanan and H. Neuberger, Nucl. Phys. B 412 (1994) 574 and B 443 (1995) 305.

[2] S. Randjbar-Daemi and J. Strathdee, Nucl. Phys. B 443 (1995) 386.

[3] S. Randjbar-Daemi and J. Strathdee, Phys. Lett. B 348 (1995) 543; Phys. Rev. D $51(1995) 6617$.

[4] R. Narayanan and H. Neuberger, Phys. Lett. B302 (1993) 62.

[5] L.H. Karsten, Phys. Lett. 104B (1981) 315;

L.H. Karsten and J. Smit, Nucl. Phys. B183 (1981) 103;

H.B. Nielsen and M. Ninomya, Nucl. Phys. B185 (1981) 20; Nucl. Phys. B193 (1981) 173; Phys. Lett. 105B (1981) 219;

J. Smit, Acta Phys. Pol. B17 (1986) 531.

For a recent review see Y. Shamir, hep-lat/9509023.

[6] M.J. Dugan and L. Randall, Nucl. Phys. B 382 (1992) 419.

[7] P.D. Swift, Phys. Lett. B 145 (1984) 256;

J. Smit, Nucl. Phys. B (Proc. suppl.) 4 (1988) 451;

I. Montvay, Phys. Lett. B 199 (1987) 89; B 205 (1988) 315.

[8] T. Kawano and Y. Kikukawa, hep-lat/9501032.

[9] D.B. Kaplan, Phys. Lett. B 288 (1992) 342.

[10] Some references to earlier work on Kaplan's domain wall approach are

H. Aoki, S. Ito, J. Nishimura and M. Oshikawa, Mod. Phys. Lett. A9 (1994) 1755;

S. Aoki and H. Hirose, Phys. Rev. D49 (1994) 2604;

S. Aoki and Y. Kikukawa, Mod. Phys. Lett. A8 (1993) 3517;

S. Chandrasekharan, Phys. Rev. D49 (1994) 1980;

M. Creutz and I. Horvath, Nucl. Phys. B(Proc. Suppl.)34 (1994) 583;

J. Distler and S-Y. Rey, PUPT-1386, hep-lat/9305026;

V. Furman and Y. Shamir, WIS-94-19-PH, hep-lat/9405004;

M.F.L. Golterman, K. Jansen and D.B. Kaplan, Phys. Lett. B301 (1993) 219;

M.F.L. Golterman, K. Jansen, D.N. Petcher and J. Vink, Phys. Rev. D49 (1944) 1606 ;

M.F.L. Golterman and Y. Shamir, WASH-U-HEP-94-61, hep-lat/9409013;

A. Hulsebos, C.P. Korthals-Altes and S. Nicolis, CPT-94-P-3036, hep-th/9406003; 
K. Jansen, Phys. Lett. B288 (1992) 348; and Desy preprint DESY-94-188, heplat/9410108;

K. Jansen and M. Schmaltz, Phys. Lett. B296 (1992) 374;

D.B. Kaplan, Nucl. Phys. B(Proc. Suppl.) 30 (1993) 597;

T. Kawano and Y. Kikukawa, KUNS-1239, hep-th/9402141;

R. Narayanan and H. Neuberger, Phys. Rev. Lett. 71 (1993) 3251; Nucl. Phys. B(Proc. Suppl.)34 (1994) 587

C.P. Korthals-Altes, S. Nicolis and J. Prades, Phys. Lett. B316 (1993) 339;

R. Narayanan, Nucl. Phys. B(Proc. Suppl.)34 (1994) 95;

Y. Shamir, Nucl. Phys. B406 (1993) 90; Nucl. Phys. B417 (1993) 167; Phys. Lett. B305 (1992) 357;

Z. Yand, Phys. Lett. B296 (1992) 151;

S. Aoki and R.B. Levien, Phys. Rev. D 51 (1995) 3790;

S. Aoki and K. Nagi, hep-lat/9508023;

M. Creutz, hep-lat/9410008; hep-lat/949411033.

[11] A. Borelli, L. Maiani, G.C. Rossi, R. Sisto and M. Testo, Nucl. Phys. B 333 (1990) 335 ;

L. Maiani, in Nucl. Phys. 29 B,C (1992) 33 (Proc. Suppl.), ed. L. Maiani et al.

[12] J.L. Alonso, Ph. Boveaud, F. Lesmes and A.J. Van der Sigs, Nucl. Phys. (Proc. Suppl.) B 42 (1995) 595 and hep-lat/95-7603.

[13] A.A. Slavnov, Phys. Lett. B 319 (1993) 231; Nucl. Phys. B 348 (1995) 553;

S.A. Frolov and A.A. Slavnoc, Nucl. Phys. B 411 (1994) 647.

[14] G. 't Hooft, Phys. Lett. B 349 (1995) 491;

A.S. Kronfeld, hep-lat/9504007;

S.D.H. Hsu, hep-lat/9503058;

W. Bretenholz and V.--J. Wise, hep-lat/9503022;

I. Montvay, hep-lat/9505015;

P. Hernandez and R. Sundrum, hep-ph/9506331.

[15] C. Itzykson, Int. J. Mod. Phys. A 1 (1986) 65.

[16] R. Narayanan, H. Neuberger and P. Vranas, Phys. Lett. B 353 (1995) 507; heplat/9507013; hep-lat/9509047; hep-lat/9509046. 